\title{
Gastrostomy in Pediatric Patients
}

\author{
Brian W. Gray, Ana Ruzic and George B. Mychaliska \\ University of Michigan, Section of Pediatric Surgery, C.S. Mott Children's Hospital \\ USA
}

\section{Introduction}

Gastrostomy is one of the most common procedures performed in the pediatric population. The first gastrostomy was performed over 150 years ago, with the first successful attempts in children occurring in the late 1800's. [1] The procedure and its use in Pediatric Surgery have significantly evolved over the past several decades with the introduction of endoscopic and laparoscopic techniques to surgical practice. [2-7] These advances have resulted in the ability to perform these operations quickly, safely, and minimally invasively, while also expanding the applications for gastrostomy.

The primary indication for gastrostomy in infants and children is the need for long-term (>3-6 months) primary or supplemental enteral feeding (Table 1). This group can be subdivided into the two most common groups of gastrostomy tube candidates: those with severe dysphagia and those with failure to thrive (FTT). Most of these children with severe dysphagia have neurologic dysfunction that impairs normal swallowing. Potential sources of FTT include short gut syndrome, GI malabsorption, malignancy, trauma, chronic lung disease, and congenital heart disease, among others. Gastrostomy may also be considered in patients with pulmonary disease due to frequent aspiration of oral feeds. Rarely, a child can require gastrostomy to administer a non-palatable diet or medication. Finally, for children with primary GI abnormalities, gastrostomy placement may be a preferred means of gastric decompression.

\begin{tabular}{|lc|}
\hline Severe Dysphagia & Frequent Aspiration (documented) \\
Neurologic Impairment & Leading to Pulmonary Disease \\
Failure to Thrive & \\
Short Gut Syndrome & Non-palatable Diet or Medication \\
GI Malabsorption & Chronic renal failure diet \\
Malignancy & HAART therapy for HIV \\
Trauma & Cholestyramine for Alagille Syndrome \\
Chronic Pulmonary Disease, e.g. CF & Gastric Decompression \\
Congenital Heart Disease & Esophageal atresia with distal TEF \\
\hline
\end{tabular}

Table 1. Indications for Gastrostomy in Children and Infants 


\section{Preoperative workup}

Prior to planning the operative approach to gastrostomy tube placement, one must assure the patient is indeed an appropriate candidate for such a procedure. Clinical indications, as well as anatomic and physiologic factors should be considered. Specifically, identifying a need for concomitant anti-reflux procedure dictates further pre-operative work-up. Practices vary, but in general, surgeons employ a combination of an upper gastrointestinal (UGI) study, $\mathrm{pH}$ probe, gastric emptying study, and in some cases endoscopy. Overuse of these resources has recently been scrutinized, largely due to poorly demonstrated utility of an UGI to evaluate GERD and therefore predict a need for an anti-reflux intervention. Consequently, a number of recent publications began advocating selective use of radiologic studies and only in cases when they were highly suspected to change the operative intervention. [8-10] In these cases, a focused history and physical exam are essential, designed to elicit symptoms of GERD and gastric feeding intolerance.

Although the sensitivity of an UGI to assess GERD is low, it remains the most utilized preoperative modality in evaluation of patients for gastrostomy tube placement. Anatomic anomalies including malrotation, delayed gastric emptying, or esophageal stricture will alter the operative plan. Clinical evidence of severe GERD, may be difficult to obtain from some patients, and precipitates additional testing including a 24-hour $\mathrm{pH}$ probe. This is particularly true for the severely neurologically impaired (NI) population, which is traditionally felt to be at high risk of symptomatic GERD. Recent literature, however, challenges this notion as well, demonstrating only a 5-7\% conversion rate to an antireflux operation, following initial gastrostomy in properly selected patients. [11,12,9] Finally, evidence of delayed gastric emptying may lead to placement of a gastro-jejunostomy tubes, allowing for gastric drainage via the gastric limb, while feeding via the jejunostomy limb.

In children with severe respiratory compromise, failure to thrive is common. These patients are some of the most frequent candidates for a gastrostomy tube, but also a population which presents a particular challenge in pre-operative planning. It is commonly unclear whether GERD precipitates and worsens their respiratory symptoms. In addition to the NI population, these children are most often submitted to additional pre-operative testing, including a 24-hour $\mathrm{pH}$ probe. Currently, the $\mathrm{pH}$ probe is considered the gold standard for establishing the diagnosis of GERD. [13] In most institutions, pediatric testing requires an overnight inpatient stay. A naso-esophageal probe is inserted, terminating approximately 2.5-3 cm above the lower esophageal sphincter. Continued measurements of the $\mathrm{pH}$ are recorded, with $\mathrm{pH} \leq 4$ indicating reflux of gastric contents. Both frequency and duration of GER are measured, as well as number of GER episodes lasting $>5$ minutes, duration of the longest episode, and percentage of time esophageal $\mathrm{pH}$ remains $\leq 4$. All of these components are then factored into a composite score (known as the DeMeester score). Values of more than 14.7 indicate pathologic GER and a need for a concomitant antireflux operation. [14]

Endoscopy is rarely used in the pediatric population solely for pre-operative evaluation prior to gastrostomy tube placement. It is a valuable tool, however, particularly when radiologic studies and history provide an inconclusive picture. Endoscopy can reliably demonstrate esophageal, gastric and duodenal ulcerations, esophagitis or gastritis, polyps, stricture or diverticula, all of which can account for feeding intolerance. Although many of these findings do not preclude placement of a gastrostomy tube, their symptoms can be identical to those of GERD. When identified, they can help establish a correct diagnosis and obviate the need for a concomitant anti-reflux procedure. 


\section{Gastrostomy techniques}

\subsection{Open/Stamm gastrostomy}

The technique for a classic open, or Stamm, gastrostomy was first described by Dr. Martin Stamm in 1894. Compared with other methods for creating a gastrostomy, the Stamm technique is being used with decreasing frequency due to its invasive nature. Indications for Stamm gastrostomy include altered anatomy, history of multiple abdominal operations, an unstable patient, and concurrent laparotomy for other reasons.

Several different small open incisions can be used for a Stamm gastrostomy. These include a small vertical incision in the midline half way between the umbilicus and the xiphoid process, a left oblique subcostal incision, or a left supraumbilical transverse incision. The subcutaneous tissue and abdominal wall is divided using electrocautery. The anterior portion of the stomach is identified and a suitable anatomic location for the gastrostomy is identified. The gastrostomy should be placed in a dependent portion of the anterior wall of the stomach near the greater curvature. The gastrostomy location should be sufficiently far away from the fundus if a subsequent fundoplication is necessary. However, the position should also be sufficiently far away from the pylorus to prevent possible pyloric obstruction from an inflated gastrostomy balloon. Two concentric purse string sutures are placed using absorbable suture. Next, a small incision is made on the abdominal wall several centimeters from the original incision. Kocher clamps are placed at the edge of the fascia and a tonsil clamp is placed retrograde through the abdominal wall. A gastrostomy tube is then pulled through the tract. Balloon and mushroom tips catheters and low-profile MIC-KEY buttons (Kimberly-Clark Worldwide, Inc) have all been used in Stamm gastrostomies. Some centers place a traditional gastrostomy tube at first, while others prefer the initial placement of a MIC-KEY button. A gastrotomy is then performed in the central portion of the double purse string sutures. The gastrostomy tube is placed in the stomach, and the balloon is inflated. The purse strings are tied down to secure the gastric serosa around the tube, and the stomach is then sutured to the anterior abdominal wall with interrupted absorbable suture in four quadrants. The small abdominal wall incision is closed with a running vicryl suture. The skin is closed with a subcuticular stitch. Dressings are applied.

\subsection{Laparoscopic gastrostomy}

Laparoscopic gastrostomy tube placement is one of the most popular methods currently used in the pediatric population, particular for smaller children. Advantages of this technique include the use of small incisions and direct visualization of the stomach upon tube insertion to avoid hollow viscus injury. A review from our institution in 2010 found a slightly lower complication rate for laparoscopic gastrostomy compared to PEG tube placement. [15] One disadvantage is that it can be challenging to bring up the stomach through the thicker abdominal wall $(>2 \mathrm{~cm}$ thick) of larger children, so laparoscopic gastrostomy tube placement is generally reserved for younger, smaller children.

To perform a laparoscopic gastrostomy, the abdomen is prepped and draped in the usual sterile fashion. Prior to insufflation, the costal margin is marked and the proposed optimal location for the gastrostomy tube in the left upper quadrant is marked. The optimal position is at least two centimeters from the costal margin in a paramedian plane. A small incision is made in the umbilicus, the abdomen is inflated with carbon dioxide, and a five millimeter trocar is inserted. A five millimeter, thirty degree laparoscope is inserted. At this time, the patient's stomach is decompressed with an orogastric tube. A small incision is made in the 
left upper quadrant in the intended position for the gastrostomy tube. Care should be taken to avoid making the incision too close to the costal margin to avoid chronic pain from the tube. A five millimeter trocar is then inserted through this incision. As in the Stamm gastrostomy, the optimal location for the gastrostomy tube is a dependent portion of the stomach near the greater curvature, sufficiently far away from the pylorus. This is grasped with an atraumatic grasper through the left upper quadrant trocar and, under direct vision, brought toward the anterior abdominal wall. The abdomen is desufflated, the trocar is removed, and a portion of the stomach brought through the small incision. A silk suture is then placed through the tip of the stomach. The stomach is then secured to the fascia in four quadrants with an absorbable suture, and a single purse string is placed using absorbable suture. A gastrotomy is performed sharply or with cautery. A gastrostomy tube is then inserted directly into the stomach, and the balloon is inflated. Again, a low-profile MIC-KEY button may be placed during the initial procedure. The abdomen is re-insufflated and the laparoscope is placed through the umbilical port to confirm proper tube position. The umbilical incision is closed with an interrupted absorbable figure-of-eight suture, and the skin is closed with a subcuticular stitch. Dressings are applied.

\subsection{Percutaneous Endoscopic Gastrostomy (PEG)}

The PEG procedure for gastrostomy placement was developed in 1980 by Dr. Michael Gauderer as a less invasive technique of feeding tube placement for children. [16] Since that time, it has been adopted worldwide as the primary method of gastrostomy placement in adults and older children. Whereas many adults can tolerate the procedure under conscious sedation, most children still require general anesthesia for PEG placement.

PEG placement begins with placing the patient in a supine position and prepping the patient's abdomen in a usual sterile fashion. Most standard PEG kits are equipped with a sterile drape that may be used to drape the abdomen. A flexible endoscope is then inserted through the patient's mouth and guided into the stomach. For most patients, a pediatric endoscope may be used, but larger children may require an adult scope. The stomach is then insufflated, but not over-inflated. An under-inflated stomach can allow the transverse colon to become interposed between the stomach and the abdominal wall, and an overinsufflation can cause inflation of the small bowel, increasing the risk of hollow viscus injury.

The surgeon at the abdomen then chooses a proper gastrostomy site under the left costal margin at least two centimeters from the margin and depresses the skin, so that the endoscopist can identify the indentation in the anterior gastric wall. The endoscopist then illuminates the anterior gastric wall along the greater curvature, and the surgeon verifies transillumination on the abdominal wall. If clear indentation transillumination cannot be attained, then another method of gastrostomy tube placement should be pursued due to the risk of hollow viscus injury. Once a site of clear transillumination is found on the abdominal wall in a proper gastrostomy site, the surgeon then infuses local anesthetic in the skin and subcutaneous tissue. A large bore needle is then inserted through the skin into the stomach. The endoscopist then advances a polypectomy snare through the endoscope and places the snare around the end of the needle. The surgeon then inserts a looped wire through the needle. This wire is enclosed in the polypectomy snare and pulled gently out through the mouth along with the endoscope. At this point, the wire marks the tract from the mouth to the future gastrostomy site. The surgeon now makes an $8-10 \mathrm{~mm}$ incision through the 
abdominal skin on either side of the wire. Next, the endoscopist attaches the steel wire loop to the tapered end of the gastrostomy tube, and the surgeon carefully pulls the abdominal end of the steel wire, gently pulling the gastrostomy tube through the mouth, esophagus, stomach, and abdominal wall. The internal gastric retainer on the gastrostomy tube should fit snugly against the anterior gastric wall. The endoscope is re-inserted into the stomach at this point to verify internal tube positioning. An external bolster and immobilizing ring are then slid over the tube to secure it into place. Care is taken to not put excessive pressure across the gastric and abdominal walls to prevent post-procedure pain and tissue necrosis. Finally, the tube is cut to the desired length, and a feeding adapter is applied to the end of the tube. No sutures are required. The PEG tube is usually changed to a low-profile button after at least six weeks to allow for tract maturation.

\subsection{Laparoscopic PEG}

Several studies have shown lower incidence of injury to the small and large bowel during laparoscopic gastrostomy compared to the PEG procedure. $[6,15,17]$ The primary reason for this difference is the direct visualization of the interface between the gastric and abdominal walls that the laparoscopic technique allows. Unfortunately, the laparoscopic gastrostomy cannot be performed on children with thicker abdominal walls. For this reason, our group is increasingly performing a laparoscopic PEG procedure. Other indications include failed attempts at a traditional PEG, altered abdominal anatomy from previous surgery, and gastrostomy placement at the conclusion of a laparoscopic operation, such as Nissen fundoplication. [4,7]

The laparoscopic PEG technique is a hybrid of the laparoscopic and percutaneous techniques. The patient's abdomen is prepped in a normal and sterile fashion, and a single five millimeter laparoscopic trocar is inserted through the umbilicus. The abdomen is moderately insufflated to allow visualization of the stomach. A standard PEG procedure as described above is then completed under direct intra-abdominal visualization to prevent hollow viscus injury. As the stomach is insufflated, the abdominal cavity is desufflated after adequate visualization. An advantage of this technique is that the gastrostomy can be placed in the ideal position on the greater curvature of the stomach between the body and the antrum. If the surgeon wishes to secure the stomach to the abdominal wall, two to four tfasteners can be placed percutaneously through the gastric wall under laparoscopic visualization. Alternatively, the surgeon has the option of suturing the gastric wall to the abdominal wall intracorporeally, but this requires placement of two additional five millimeter ports. We have not found this to be necessary in our experience. As with PEG tubes in children, laparoscopic PEGs are usually changed to a low-profile button in a separate procedure at least six weeks after initial placement.

\subsection{Image-guided percutaneous gastrostomy}

A recent advance in minimally invasive gastrostomy is the percutaneous placement of a gastrostomy tube under fluoroscopic guidance. This technique is performed by interventional radiologists or surgeons with advanced training in interventional radiology. The ideal patient is one without complex abdominal anatomy or previous abdominal surgery. The technique involves insufflating the stomach using an NG tube under fluoroscopic visualization. A needle is then advanced into the stomach using fluoroscopy, with entry into the lumen verified by contrast injection. A t-fastener is advanced through the 
needle to keep the stomach adhered to the anterior abdominal wall. A total of two to four tfasteners are used to secure the stomach. A needle is then advanced into the stomach through the center of the t-fasteners, and its location is verified by contrast injection. Next, a wire is advanced through the needle into the stomach, and the tract is dilated using the Seldinger technique. Once the tract is sufficiently dilated, a pigtail or balloon type gastrostomy tube is advanced into the stomach, and positioning is again verified by contrast injection. Finally, the t-fasteners are tied externally over bolsters. These fasteners can be cut between three and seven days post-procedure. As with other gastrostomy tubes, feeding is usually initiated within twenty-four hours of tube placement. One large study comparing outcomes between PEG and image-guided gastrostomy found low rates of major complications in both groups, but significantly less complications in the PEG group. [18] This indicates that image-guided percutaneous gastrostomy should not be the standard of care for feeding tube placement at this time.

\section{Postoperative care}

Feeding through a new gastrostomy tube may begin within the first twelve to twenty-four hours after placement, barring other complicating factors, such as postoperative ileus after larger operations. Tube feeds are initiated slowly and then advanced to goal rate by the second postoperative day, or as tolerated by the patient. It is important to keep the patient's skin clean and dry by preventing leakage of gastric contents onto the skin. This can be done by keeping the external bolster snug, but not too tight, against the skin and securing the tube at a perpendicular angle to the abdominal wall. Keeping the tube perpendicular at the skin decreases the amount of soft tissue stretching encountered at the gastrostomy site. Finally, the area around the gastrostomy tube should be cleansed on a daily basis with soap and water starting forty-eight hours after surgery. These children should receive close follow-up after gastrostomy placement due to the risk of several catheter-related complications.

\section{Complications}

Although a common pediatric surgery procedure, gastrostomy tube placement can have serious associated complications. Early recognition, aided by a high index of suspicion, allows for prompt intervention and prevention of catastrophic sequelae. Intraoperative, early postoperative, and remote complications have all been described. [19] Knowing their timeline, as well as presentation, guides further investigations and therapy. Table 2 outlines the specific complications and the timeline in which they generally occur.

Regardless of the operative technique, hollow viscus injury, liver injury, bleeding, and malposition of the tube can occur during the creation of gastrostomy. If recognized at the time of the operation, they can be addressed immediately, preventing serious post-operative complications, including hemodynamic instability, peritonitis, and sepsis. Early postoperative complications, however, have a more subtle presentation and require a high index of suspicion. Often they are not recognized until initiation of feeds. Their symptoms vary and range from early feeding intolerance and ileus, to worsening abdominal pain, peritonitis, and signs of systemic infection. Abdominal X-rays, G-tube studies, and UGI studies can show pneumoperitoneum suggesting separation of the stomach from the abdominal wall, intraperitoneal leak from tube dislodgment, and small or large bowel 
injury. Contrast studies are also useful in demonstrating appropriate intra-luminal position of tube, gastric outlet obstruction from tube migration, and gastro-colonic fistulae. Some studies have shown laparoscopic placement to be associated with less complications than PEG because of direct visualization of the stomach and greater ability to secure the stomach to the anterior abdominal wall. [15]

Less severe remote complications result in frequent outpatient evaluations following the initial post-operative visit. Granulation tissue formation, tube erosion, and skin ulceration produce significant discomfort for the child, cause bleeding and leakage of the gastric contents, and lead to frequent interruption of feeds. Short of resiting the gastrostomy, the most efficient treatment of these complications is prevention. Meticulous hygiene and appropriate positioning of external tubing prevents stretching of the skin and subcutaneous tissues and subsequent enlargement of the gastrostomy tract. Parental education, both preoperatively and post-operatively, is essential in preventing many of the skin and tract complications.

\begin{tabular}{|ll|}
\hline Intraoperative Complications & Remote complications \\
Hollow viscus injury & Granulation tissue formation \\
Liver injury & Tube erosion \\
Colonic placement (PEGs) & Skin ulceration \\
Bleeding & Intraperitoneal leak following tube \\
Early Postoperative Complications (1-4 weeks) & Gastro-colonic fistula \\
Early tube dislodgment & Gastro-cutaneous fistula \\
Gastric separation from the abdominal wall & \\
Intra-peritoneal leak & \\
Tube occlusion & \\
Gastric outlet obstruction & \\
Surgical site infection & \\
\hline
\end{tabular}

Table 2. Common complications of gastrostomy tube placement

\section{Conclusion}

Gastrostomy tube placement in pediatric patients is a time-tested procedure that has allowed countless children the ability to attain sufficient caloric intake and thus promote healing and growth. The decision to place a gastrostomy tube is based primarily on clinical factors with ancillary testing as needed. In most patients, the tube can be removed once they demonstrate adequate oral consumption of calories to sustain continued growth and development. Once removed, the ability for the gastrostomy tract to close spontaneously appears to be dependent on the amount of time the tube was in place. One study found that tubes left in place longer than 8 months had a significantly higher chance of leaving a gastrocutaneous fistula after the gastrostomy tube was removed. [20] Some children, particularly the neurologically impaired, require an enteral feeding tube indefinitely. Advances in endoscopy and laparoscopic surgery have increased the ease of tube placement, minimized invasiveness, and decreased morbidity. 


\section{References}

[1] Gauderer, M.W. and T.A. Stellato, Gastrostomies: evolution, techniques, indications, and complications. Curr Probl Surg, 1986. 23(9): p. 657-719.

[2] Jones, V.S., E.R. La Hei, and A. Shun, Laparoscopic gastrostomy: the preferred method of gastrostomy in children. Pediatr Surg Int, 2007. 23(11): p. 1085-9.

[3] Gauderer, M.W., Percutaneous endoscopic gastrostomy-20 years later: a historical perspective. J Pediatr Surg, 2001. 36(1): p. 217-9.

[4] Charlesworth, P., M. Hallows, and A. van der Avoirt, Single-center experience of laparoscopically assisted percutaneous endoscopic gastrostomy placement. J Laparoendosc Adv Surg Tech A. 20(1): p. 73-5.

[5] Georgeson, K.E., Laparoscopic gastrostomy and fundoplication. Pediatr Ann, 1993. 22(11): p. 675-7.

[6] Zamakhshary, M., et al., Laparoscopic vs percutaneous endoscopic gastrostomy tube insertion: a new pediatric gold standard? J Pediatr Surg, 2005. 40(5): p. 859-62.

[7] $\mathrm{Yu}$, S.C., et al., Laparoscopic-assisted percutaneous endoscopic gastrostomy in children and adolescents. JSLS, 2005. 9(3): p. 302-4.

[8] Valusek, P.A., et al., Does an upper gastrointestinal study change operative management for gastroesophageal reflux. J. Pediatr Surg, 2010. 45: p. 1169-1172.

[9] Wheatley, M.J., et al., Long-term Follow-up of Brain-Damaged Children Requiring Feeding Gastrostomy: Should an Antireflux Procedure Always Be Performed? J Pediatr Surg, 1991. 26(3): p. 301-305

[10] Cuenca, A.G., et al., The Usefulness of the Upper Gastrointestinal Series in the Pediatric Patient Before Anti-Reflux Procedure or Gastrostomy Tube Placement. J Surg Res, 2011. P 1-6 (Epub ahead of print) http:/ / www.ncbi.nlm.nih.gov/pubmed/21550057

[11] Novotny, N.M, A.L Jester, and A.P. Ladd, Preoperative prediction of need for fundoplication before gastrostomy tube placement in children. J Pediatr Surg, 2009. 44: p. 173-177.

[12] Kawahara, H., et al., Should fundoplication be added at the time of gastrostomy placement in patients who are neurologically impaired? J Pediatr Surg, 2010. 45: p. 2373-2376

[13] Soares, R.V., et al., Interstitial Lung Disease and Gastroesophageal Reflux Disease: key role of esophageal function tests in the diagnosis and treatment. Arq Gastroenterol, 2011. 48(2): p. 91-97.

[14] Wang, J., et al., Composite score of reflux symptoms in diagnosis of gastroesophageal reflux disease. World J Gastroenterol, 2004. 10(22): p. 3332-3335.

[15] Akay, B., et al., Gastrostomy tube placement in infants and children: is there a preferred technique? J Pediatr Surg, 2010. 45(6): p. 1147-52.

[16] Gauderer, M.W., J.L. Ponsky, and R.J. Izant, Jr., Gastrostomy without laparotomy: a percutaneous endoscopic technique. J Pediatr Surg, 1980. 15(6): p. 872-5.

[17] Lantz, M., H. Hultin Larsson, and E. Arnbjornsson, Literature review comparing laparoscopic and percutaneous endoscopic gastrostomies in a pediatric population. Int $\mathrm{J}$ Pediatr. 2010: p. 507616.

[18] Nah, S.A., et al., Gastrostomy insertion in children: percutaneous endoscopic or percutaneous image-guided? J Pediatr Surg. 45(6): p. 1153-8.

[19] Fortunato, J.E and Cuffari, C. Outcomes of Percutaneous Endoscopy Gastrostomy in Children. Curr Gastroenterol Rep 2011. 13: p. 293-299

[20] Gordon, J.M. and J.C. Langer, Gastrocutaneous fistula in children after removal of gastrostomy tube: incidence and predictive factors. J Pediatr Surg, 1999. 34(9): p. 1345-6. 


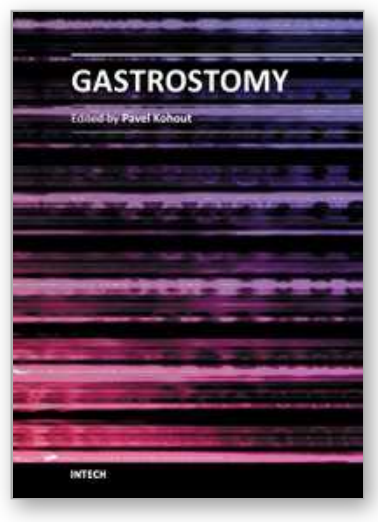

\author{
Gastrostomy \\ Edited by Dr. Pavel Kohout
}

ISBN 978-953-307-365-1

Hard cover, 152 pages

Publisher InTech

Published online 02, December, 2011

Published in print edition December, 2011

The gastrostomy placement is a method of providing nutrition to the patients who are unable to eat. In this book you can find chapters focused on the use of gastrostomy in children, patients with neurological impairment and patients with head and neck tumours. Home enteral nutrition is suitable for all of these groups of patients and is far easier with gastrostomy. The new indications (especially in very young children) required new techniques such as: laparoscopic gastrostomy, laparoscopy assisted endoscopic gastrostomy with/without fundoplication, ultrasonography assisted gastronomy. All information about these techniques can be found in this book. This book does not serve as a basic textbook, but as an interesting reading material and as an aid for physicians who are already familiar with the indication for gastrostomy and want to know more.

\title{
How to reference
}

In order to correctly reference this scholarly work, feel free to copy and paste the following:

Brian W. Gray, Ana Ruzic and George B. Mychaliska (2011). Gastrostomy in Pediatric Patients, Gastrostomy, Dr. Pavel Kohout (Ed.), ISBN: 978-953-307-365-1, InTech, Available from:

http://www.intechopen.com/books/gastrostomy/gastrostomy-in-pediatric-patients

\section{INTECH}

open science | open minds

\section{InTech Europe}

University Campus STeP Ri

Slavka Krautzeka 83/A

51000 Rijeka, Croatia

Phone: +385 (51) 770447

Fax: +385 (51) 686166

www.intechopen.com

\section{InTech China}

Unit 405, Office Block, Hotel Equatorial Shanghai

No.65, Yan An Road (West), Shanghai, 200040, China

中国上海市延安西路65号上海国际贵都大饭店办公楼405单元

Phone: +86-21-62489820

Fax: $+86-21-62489821$ 
(C) 2011 The Author(s). Licensee IntechOpen. This is an open access article distributed under the terms of the Creative Commons Attribution 3.0 License, which permits unrestricted use, distribution, and reproduction in any medium, provided the original work is properly cited. 